the chemist, who found lead in them, and testified that the subjects died of lead-poisoning. The newspaper reports say, " The evidence was conclusive that several members of the Diebel and Palmer families died of lead-poisoning." The verdict of the jury was, that "the deaths of the four persons were undoubtedly due to chronic lead-poisoning, and that the poisoning resulted from the use of chromate of lead as a coloring-matter in buns and other breadstuffs." The only allusion to chrome-salts in the entire reports, as given in two newspapers, was made by Dr. Leffman, who said, "The traces of chromium had disappeared." We have here four cases well authenticated, in which lead chromate produced death, - produced it by chronic lead-poisoning, and not by any action of the chromium trioxide present.

The theory of antidotes, as understood by this writer, is that substances insoluble in any of the juices of the animal body are harmless as poisons. If we do not accept this as a fundamental proposition, it is difficult to see how we reasonably can employ any antidote supposed to act upon that principle. Chromic acid is a very active oxidizer. In contact with organic matter, it is quickly reduced to chromic oxide (a compound insoluble in any of the juices of the animal body). It is a destroyer of organic tissues, therefore. The action of both normal and acid alkali chromates is similar to chromic acid. They destroy organic matter by oxidizing it, chromic oxide being precipitated. Chromic acid and soluble chromates are then poisonous in the same sense as are sulphuric acid and nitric acid. Chromic oxide is harmless.

Concerning the Philadelphia cases mentioned, the writer cannot even pretend to speak with any authority. Any one, however, familiar with the oxidizing action of chromic-acid salts, and who is accustomed to making combustions with lead chromate, would not find much difficulty in believing that the small quantity of lead chromate taken by any one victim was reduced while in contact with organic matter in the stomach and intestines, chromic oxide passing out with the dejecta, and lead oxide being left to produce its cumulative poisonous effects.

When one states that the insoluble modifications of chromium are in any way poisonous to the animal body, the burden of proof rests upon him. The soluble salts (the alkali chromates) do, however, produce sores and sloughing under certain conditions: (I) if they fall in quantities upon an abrasion of the skin, or (2) upon the delicate mucous membrane. Speaking generally, a solution containing I 50 grams of an alkali chromate in one litre of water is scarcely strong enough to produce sores upon the hands. Again, generally, alkali chromate dust which is just visible without direct sunlight is harmless, unless one should remain in it for some weeks. When such dust falls upon the mucous membrane, it is quickly reduced by the secretion it finds there, and chromic oxide is precipitated. The membrane is not attacked. There can be no doubt that Dr. Harrington found in the clothing he examined, the large quantities of chromium salts mentioned by him. It would have been rather strange if he had not, since most cloth is chrome-dyed, and contains about eight-tenths of one per cent chromic oxide in its fibre. Had he examined further, equally certain, he would have found iron, cellulose, keratin, and some other organic products. Why not assign to one or all of them the maladies of the patients mentioned? So far as his paper gives evidence, or so far as I know, cellulose is equally as poisonous as the insoluble chrome-dye on yarn. We should risk little in saying this is true so far as any one knows. Before we say otherwise, we should offer such proofs as would lead a cautious man to accept the statements.

To those who read the communication alluded to, it may be of interest to know that a suit of clothing for a small boy will lose in weight about three hundred and forty milligrams in a week. Of this, about eight-tenths of one per cent is chromic oxide, when the clothing is chrome-dyed. Such clothing then loses about two and seven-tenths milligrams of chromic oxide in one week. How much of this latter the boy would be likely to inhale as dust, and what injury it would do him, are matters about which I have no information to offer. If it were arsenic, and he inhaled the whole of it in one day, and twenty times as much more, it might possibly sicken him.

Five hundred milligrams of the chrome-dyed cotton cloth before mentioned was ground between the teeth of a healthy man, and slowly swallowed. It produced no effect which could be detected. This cloth contained four milligrams of chromic oxide. Such a small quantity was taken only because of the desire to test the effects of minute quantities. The only inconvenience resulting from an attempt to eat a yard square of such cloth would be in masticating it. Even between strong molars, it is really difficult to grind.

To sum up, finally, the writer has not been able to show that chrome-dye, or indeed any modification of chromium, is in any way poisonous to the human body. The continuation of these studies is left to others better fitted to pursue them, and with the sincere hope that the subject will not be allowed to die of neglect. The matter intimately concerns the general welfare. WM. GLENN.

Baltimore, July $2 \mathbf{r .}$

\section{Distillery-Swill as a Food for Milch-Cows.}

THE interest in this subject, developed by the recent discussion in Science, prompts me to give its readers some additional points of interest. Until within the past four years, the practice of feeding distillery-waste on Long Island was very prevalent. Although the ordinances of the city of Brooklyn have for years forbidden the use of this food, no systematic effort was made to stamp it out previous to the year r883. At that time it was used very generally in Brooklyn and its suburbs, especially during the winter months. Since then it has been almost entirely discontinued through the efforts of the Health Department.

It is the almost unanimous opinion of cow-keepers who have fed swill and dry feed, that the cows do better without swill; by which they mean that they are healthier and less subject to diseases. This statement is of value, as it is the result of a practical trial extending over several years of time, and under conditions otherwise the same. It is the verdict of a large number of stables where the experiment has been tried by different men. To me, this testimony is conclusive as to the question of this food upon the health of the cows fed upon it. Tuberculosis and pleuro-pneumonia are more prevalent in stables where swill is fed than where 'grains,' meal, and hay are fed. This statement is based upon personal observation, and the testimony of the dairymen themselves.

A word as to the manner of feeding swill. The cows do not take kindly to this food, and must be starved to it, as a rule. Consequently, when it is to be fed, it is necessary to withhold other food, for a time at least. I have rarely seen it mixed with other food, and, in fact, the testimony of milkmen in this vicinity is that it is next to impossible to get cows to eat a mixture of swill and other food, excepting hay. The digestion of cows fed upon swill soon suffers derangements that prevent their eating such other food. Whenever it is fed at all, it is claimed that it must constitute almost the only food, - and thus it was fed in this vicinity, - when fed at all. As all advocates of swill as food for milch-cows seem to make their advocacy rest upon a liberal supply of other food with it, it becomes an important point to determine whether cows will eat hot swill, and cold meal or grain, at the same time. The experience of Long Island dairymen seems to indicate that success in this direction is doubtful, and prejudicial to the supply of milk as well as to the health of cows.

Statements have been made from time to time that distillerywaste contains alcohol and fusel oil, and that these alcohols have a bad effect upon the animals. This is an error. Both alcohol and fusel oil are separated from the waste as completely as possible; and my analyses have failed to reveal more than mere traces of either.

Sensational statements have been made that the tails of cattle fed upon this food atrophy and fall off. This statement is based upon the results of anti-pleuropneumonic inoculation seen in such stables. From the prevalence of pleuro-pneumonia among cattle fed upon this hot feed, the owners have for years resorted to a clumsy method of inoculation, in the tail, with a slice of diseased lung. In a considerable number of cases, septicæmia results, necessitating an amputation of the tail. The loss of the tails has nothing to do with the food.

When cows are kept most of the time in stalls, and fed upon this hot food, they become feverish. The temperature usually ranges from IOI. $5^{\circ} \mathrm{F}$. to $102.5^{\circ} \mathrm{F}$. This must be regarded as an 
unhealthy temperature, and no cow that has a persistent temperature of $102^{\circ} \mathrm{F}$. can give a wholesome milk.

The conditions and methods here set forth are those that almost universally prevailed on Long Island when this food was used; and the milk, which I have analyzed, and which was referred to in previous numbers of this journal, was produced under these conditions.

The chemical features of the milk which are most marked, are, the strongly acid re-action, deficiency of fat and sugar, and great excess of caseine or curd. So marked were these features, that I was able to identify swill-milk produced under these conditions.

That such milk is a dangerous food for young children, I have had abundant clinical evidence. The tough, hard curd produced in the stomach by this milk is entirely too much for the digestive powers of even healthy children, and passes undigested, irritating the intestinal mucous membrane throughout its entire length, giving rise to intestinal catarrh, inflammatory diarrhœea, or cholera-infantum. Occasionally the curd formed in the stomach is too large and firm to pass the pyloris, when the child passes into rapid collapse and death, unable to rid the stomach of the mass either by vomiting or purging. Remedies, under such circumstances, are of no avail. I have seen the same effects, in a somewhat less degree, produced in adults who were not disturbed by a pure, wholesome milk.

I think we may accept it as demonstrated, Ist, that fed as it has always been in this vicinity, distillery-swill is an unfit food for milch-cows, as it deteriorates the health of the cows, and produces unwholesome milk; 2 d, from the difficulty of perfectly controlling the manner of feeding, it is not warrantable for any sanitary authority to recommend it as a food for milch-cows ; 3 d, it has not yet been proven that it can be successfully fed in conjunction with other wholesome food; 4 th, the laws now in force in this and other States, forbidding the sale of swill-milk, are justifiable, and should be enforced.

Brooklyn, July 23.

E. H. BARTLEy, M.D.

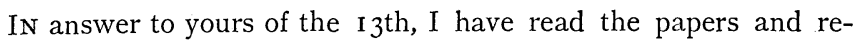
ports on distillery-swill milk published in Science, and, in my judgment, you have furnished reasonable evidence of the unwholesomeness of such milk as a general fact. I mean where distillery-swill constitutes the chief food of the animals.

From the very nature of the case, complete proof and absolute demonstration by direct, full, and conclusive experiments cannot be had : so we must rely on such evidence as is available, the same as in so many other sanitary questions where the deductions have to be derived from a great mass of cumulative evidence, each single fact in which is inconclusive. I have therefore only to suggest that you continue the recording of facts as the only available way either of arriving at just conclusions or of convincing the public of the truthfulness of the conclusions.

I know of no conclusion in sanitation so well established that men may not be found to deny it; and, so long as distillery-swill milk does not kill or sicken all who may use any of it, there will probably be persons who deny that it is harmful to any.

The correspondent in Science of July 22 (p. 46) fails to find "positive evidence" of " any ill effects of swill upon cows fed with it." On the other hand, he asserts, as if on "positive evidence," that " the evils attributable to it are largely, if not entirely, to be ascribed to the unsanitary surroundings of the animals." I hardly know how comprehensive and sweeping he intends this to apply. It would be unfair to charge him with asserting that the almost universal disease in distillery stables, the emaciation, the lax bowels, the loss of teeth, the short lives in such stables as distinguished from those where hay and grain are the chief food, and the "unsanitary surroundings of the animals" in these stables, as a rule, should be merely curious coincidences, and not due to the feeding of the swill itself. His language implies all this, but surely I can hardly believe that to be his meaning.

Some supplementary statements, however, are equally positive and equally striking, - the proposition " that lactation in a dairy is not a normal process," and that he regards the conclusions (if not, indeed, the facts) given by certain chemists and physicians, regarding the nature and digestibility of the curd of swill-milk, as on the whole unworthy of confidence.

If you can convince a.few orphan-asylums and foundling-hospitals that it would be an innocent and harmless experiment to feed half of their children on distillery-swill milk, and the other on grass-andgrain milk, and continue this experiment for several years, on different races of children, in different localities, some of the swill-milk stables to be kept as clean as other stables may be, by some process not yet announced, and carefully record and collate all the results, the question would then be settled, in the usual acceptance of that term.

Until some such plan for " positive evidence" be secured, I suggest that you work at the method of cumulative evidence which has been so rich in conclusions and beneficent in its results in other departments of sanitary science.

New Haven, Conn., July 25.

\section{State Interference.}

Copies of Science containing two of the articles on State interference have been received; also your note asking opinions respecting them.

I am glad you undertook the investigation, and wish it might have called forth more elaborate replies. It is a subject which ought to be worked up carefully for all the States and for a period of years long enough to show the working of tendencies.

But, so far as the facts you have presented go, I see very little which will not be found upon the statute-books of England, which is generally known as the classic land of laissez faire. The protective system involves more serious interference with private concerns than almost any of the new laws. Our legislators, I admit, are ignorant, and moved largely by party or private interest; but that is the fault of our political system, and is connected with the essentially commercial character of the people. The social question is upon us: we must have laws regulating competition to a certain extent. The danger comes, not from the tendency toward such regulation, but from the character of the men to whom legislation is intrusted: hence the necessity of civil-service reform, of higher political education, and of a strengthening of the moral tone of the people.

Short Beach, Conn., July 23.

H. L. OSGOOD.

\section{Tornado Force.}

MR. E. B. GARRIATT has a communication on tornado power in your issue of July 22, in which he complains of the "disposition, on the part of writers on scientific subjects, ... to sacrifice common-sense reasoning and probable facts to profound but improbable theories," and then proceeds to explain tornado energy as due to electricity, and not to wind. To support this view, he makes the statement, first, that "moist air is one of the best known conductors of electricity;" and, second, that the "earth is the great reservoir for the electric fluid." It might be worth the while for Mr. Garriatt to assure himself of the truth of his fundamental principles before he applies them on so large a scale. There is not the slightest experimental evidence that moist air is a conductor at all, much less "one of the best;" and as for the earth being a reservoir of electricity, every thing that is known about electricity negatives the idea.

Again : it is implied that electrical energy is more destructive than other kinds, as if a definite quantity of it could do more work than an equal quantity of other energy. He also speaks of "the electric fluid." These quotations show that he has no practical acquaintance with what physicists call 'electricity ;' that he does not understand the laws of its generation, the conditions of its transferrence, nor its quantitative relation to other forms of energy; and therefore, to quote still further from his article, it is " unsatisfactory and worthless from a practical scientific standpoint."

College Hill, Mass., July 23 .

A. E. Dolbear.

\section{Answers.}

ro. Robin's NEST. - The ten-storied robin's nest mentioned in Science of July 22 is indeed a remarkable affair. It is rather unusual for robins to build a new nest on an old one, although it sometimes happens. I have seen a number of two-storied nests, and one three-storied one, but such nests are rare. J. A. AllEN. - New York, July 26. 